\title{
Development of the Orbital-Free Density Functional Approach: The Problem of Angles between Covalent Bonds
}

\author{
Victor G. Zavodinsky¹, Olga A. Gorkusha ${ }^{2}$ \\ ${ }^{1}$ Institute for Material Science, Khabarovsk, Russia \\ ${ }^{2}$ Institute of Applied Mathematics, Khabarovsk, Russia \\ Email:vzavod@mail.ru, o_garok@rambler.ru
}

Received 4 January 2016; accepted 25 March 2016; published 28 March 2016

Copyright (C) 2016 by authors and Scientific Research Publishing Inc.

This work is licensed under the Creative Commons Attribution International License (CC BY).

http://creativecommons.org/licenses/by/4.0/

\section{(c) (i) Open Access}

\begin{abstract}
The Paulie's principle is used for development of the orbital-free (OF) version of the density functional theory. On the example of the three-atomic clusters, $\mathrm{Al}_{3}, \mathrm{Si}_{3}$, and $\mathrm{C}_{3}$, it is shown that the oF approach may lead to equilibrium configurations of atomic systems with both the metallic and covalent bonding. The equilibrium interatomic distances, interbonding angles and binding energies are found in good accordance with the known data. Results will be useful for developing of theoretical study of huge molecules and nanoparticles.
\end{abstract}

\section{Keywords}

Orbital-Free, Density Functional, Covalent Bonding, Angular Bond Dependence

\section{Introduction}

A number of works (for instance [1]-[9]) devoted to development of the orbital-free (OF) version of the density functional theory (DFT) [10] [11] appeared last years. This approach, in contrary to the Kohn-Sham (KS) method, does not use the wave functions (orbitals) and operates with the only electron density. The OF approach is a consecutive development of the idea of Hohenberg-Kohn [11] that the basic state of a quantum system can be completely described by means of electronic density.

There is a significant progress in modeling of two-atomic systems [3] [8] and even simple crystals [12]. However, even now there is a big unsolved problem interfering further development of the OF approach. This problem concerns description of the angular dependence of the interatomic bonding in systems with covalent bonds. As we know, till now there are no works where this problem is solved awfully. 
An essential trouble is the fact that the electronic density of a single (isolated) atom is spherical, i.e. the "orbital-free" atom has a shape of a ball, but balls form the close packed structures. For example, three identical atoms are obliged to form an equilateral triangle with corners of 60 degrees. At the same time, it is known that three atoms of silicon form an isosceles triangle with the main corner of about 80 degrees [13], atoms of carbon build a linear chain [14], and atoms of aluminum really behave like balls - they form a correct equilateral triangle [15]. Obviously, if an approach can describe the angular properties of trimers, it can solve the same problem for more complicated atomic systems too.

In our recent papers [16] [17], we have showed how it is possible, using single-atoms calculations by the Kohn-Sham DFT method (KS-DFT), to find the kinetic functions for single atoms and to use them for orbital-free modeling of atomic dimers. The present work is an attempt to develop (on example of $\mathrm{Al}$, Si, and $\mathrm{C}$ trimers) a technique which would allow to describe an adequate geometry of interatomic bonds in polyatomic systems in the framework of the OF approach.

\section{A Principal Point and Results}

As in our recent works we used here the pseudopotential approach with the FHI98pp package [18] as a generator of pseudo-potentials and equilibrium partial electron densities for single atoms. The general way of calculations is the same as described in [18]. Studied atoms were located in a cubic cell of the $L$ size $(L=52$ a.u.; 1 a.u. $=$ $0.529 \AA$ ). The cell was divided on $128 \times 128 \times 128$ elementary sub-cells for the integration with the step $\Delta L$ of 0.406 a.u. Results of calculations were compared with published data.

Transfer from dimers to trimers is a principal point because trimers characterized by some angles between atomic bonds.

To describe the angular dependence of interatomic bonding in the framework of the OF approach we must analyze the reasons of this dependence in the standard quantum-mechanical approach, which uses wave functions and electronic states. For example, it is specified in the work [19] that the angular peculiarities of a cluster $\mathrm{Si}_{3}$ are defined by the Yang-Teller effect caused by existence of the energy gap between occupied and empty states. In other words, the difference of structures of semiconductor and metal small clusters is connected with the difference of their electronic states and bond wave functions. Namely, covalent atoms have full occupied bonding states corresponded to localized functions orientated between nearest atoms; these states are separated by the energy gap from above lied non-occupied states. Metallic atoms have dispersed functions without orientation in the space and without energy gaps between them.

In our case wave functions are absent, electronic states are absent too, and, therefore, we cannot speak about any energy gap. In the OF approach we deal only with the electronic density which defines all energy and structure of the polyatomic system. However the main quantum-mechanical rules remain fair and in this case. We have not to forget that quantum mechanics is not only a set of wave functions and electron states. We have not to forget the Paulie's principle specifying that in one quantum state there can be only two electrons (without taking into account a spin). In our case this principle may be paraphrased by a following way: a covalent bond is formed by two electrons, the common wave function of which is localized in the space between two nearest atoms. It is obvious that the quantity of the electrons which are responsible for this bond doesn't change as the distance between atoms changes (if, of course, the bond isn't broken at all and the electronic structure isn't reconstructed completely). In case of the metals the states are close each other and electrons can easily "flow" from one state to another during the changing of the atomic geometry.

The above mentions may be reformulated in the language of the electronic density: the density integral $\left(n_{\text {int }}\right)$ between atoms with covalent bonding, has to remain its value at change of distance between atoms; in case of metal bonding the value of the integral $n_{\text {int }}$ can be any possible. Certainly, there is a question: on what space we have to provide integration? And what have we to do with intermediate cases, with atoms of different types? We will leave these questions for the future, and now we will try to explain the difference in structure of covalent and metal systems on the example of homogeneous clusters $\mathrm{Al}_{3}, \mathrm{Si}_{3}$ and $\mathrm{C}_{3}$.

It is obvious that the space of integration has to be rather local and at the same time it has to give us the information on quantity of the electrons included in a covalent bond. In the present work we used the space having a shape of the slab situated between two nearest atoms (Figure 1) and oriented perpendicularly to the plane in which the trimer triangle is placed. Thickness of a slab was taken of $2 \Delta L$, but for some test calculations we used slabs of $4 \Delta L$ and $8 \Delta L$ thickness. As the number of the integration points can be changed at change of the trimer configuration, the value of $n_{\text {int }}$ was normalized to one point. 


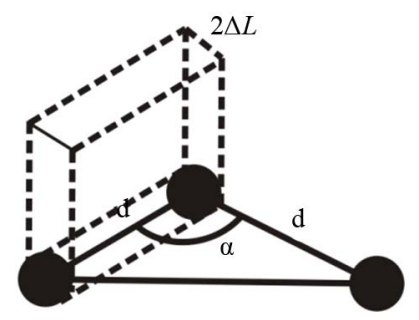

Figure 1. The scheme of an arrangement of atoms in a trimer. Dashed lines show the space on which the electronic density is integrated for definition the number of the electrons involved in the covalent bond; $\alpha$ is a corner between bonds with identical lengths of $d$.

As it was said above the integral of electron density between the nearest atoms is the critical parameter for covalent bonding. It cannot be larger than some value that is a characteristic of a so called saturated bond. Let us take the interatomic bond in a dimer as a standard saturated bond. Let us call the value $P=\frac{n_{\mathrm{int}}^{\text {trimer }}}{n_{\mathrm{int}}^{\text {dimer }}}$ (the relation of $n_{\text {int }}$ of a trimer to $n_{\text {int }}$ of a dimer) as "the bonding strength" and accept that for covalent bonds the value of $P$ shouldn't exceed 1.0 at change of the distance between atoms. For metallic bonds the value of $P$ can be any possible.

Calculated values $\mathrm{P}$ for clusters $\mathrm{Al}_{3}, \mathrm{Si}_{3}$, and $\mathrm{C}_{3}$ are presented in Figure 2 as functions of the angle between interatomic bonds in the case when restrictions on these values are absent. For each angle we found the values of interatomic distances, which corresponded to the minimum of the total energy of the cluster. One can see that $P$ is equal approximately 1.0 at $\alpha=180^{\circ}$ and increases when $\alpha$ approaches to $60^{\circ}$. The maximum value $(P=1.40)$ is observed for carbon, that has the smallest interatomic distances. Interatomic distances in aluminum and silicon are approximately the same, therefore no wonder, as the "bonding strengths" for the $\mathrm{Al}_{3}$ and $\mathrm{Si}_{3}$ trimers are approximately equal.

In order to check reliability of our results we repeated the calculations using thicker slabs $(4 \Delta L$ and $8 \Delta L)$ for integration of values $n_{\text {int }}$ (dimer) and $n_{\text {int }}$ (trimer) between atoms in dimers and trimers and we found that results have changed no more than by 2 per cent.

In Figure 3 (curve A) we present results of calculations of binding energy for the $\mathrm{Al}_{3}, \mathrm{Si}_{3}$ and $\mathrm{C}_{3}$ trimers without restriction of the "bonding strengths". From these curves it is clear that in all three cases the minimums of the binding energy correspond to triangular clusters, "bonding strengths "in which significantly exceed the corresponding values, characteristic for linear chains. This result looks natural for aluminum as its states have the metallic, not localized character; but for the clusters of $\mathrm{Si}_{3}$ and $\mathrm{C}_{3}$ having covalent bonds it is necessary to introduce the restrictions on values of $P$ stipulated above. We have taken into account this condition $(P=1)$ and found dependences of the binding energy on the angle between bonds in the $\mathrm{Si}_{3}$ and $\mathrm{C}_{3}$ clusters (Figure 3, curve $B$ ). One can see that atoms of carbon seek to form linear chains, while for silicon neither a linear chain, nor an equilateral triangle is energetically favorable: atoms of silicon prefer to form an isosceles triangle with the angle $\alpha$ of 80 degrees.

Equilibrium values of interatomic distances $d$, angles $\alpha$, and binding energies $E_{b}$ (absolute values) for the trimers $\mathrm{Al}_{3}, \mathrm{Si}_{3}$, and $\mathrm{C}_{3}$ are collected in Table 1 (calculated for the condition $P=1$ ) in comparison with known data. One can see that comparison is good. Thus, we showed that orbital-free approach is capable to describe correctly orientations of interatomic bonds in atomic clusters, as well as values of interatomic distances and binding energies.

It is interesting to compare our results for interatomic densities with results of standard KS-DFT calculations. For this purpose we calculated "bonding strengths" $\mathrm{P}$ for $\mathrm{Si}_{3}$ and $\mathrm{Al}_{3}$ using the popular KS-DFT code FHI96md [28] for the same triangles as were studied above. We have found that $\mathrm{P}$ was equal $1.0( \pm 0.02)$ for all cases for $\mathrm{Si}_{3}$, but it increased up to 1.3 for the $\mathrm{Al}_{3}$ equilateral triangle. These results are in excellent agreement with our OF ones.

\section{Conclusions}

We showed that the use of the restriction principle for the interatomic density (following from the Paulie's 


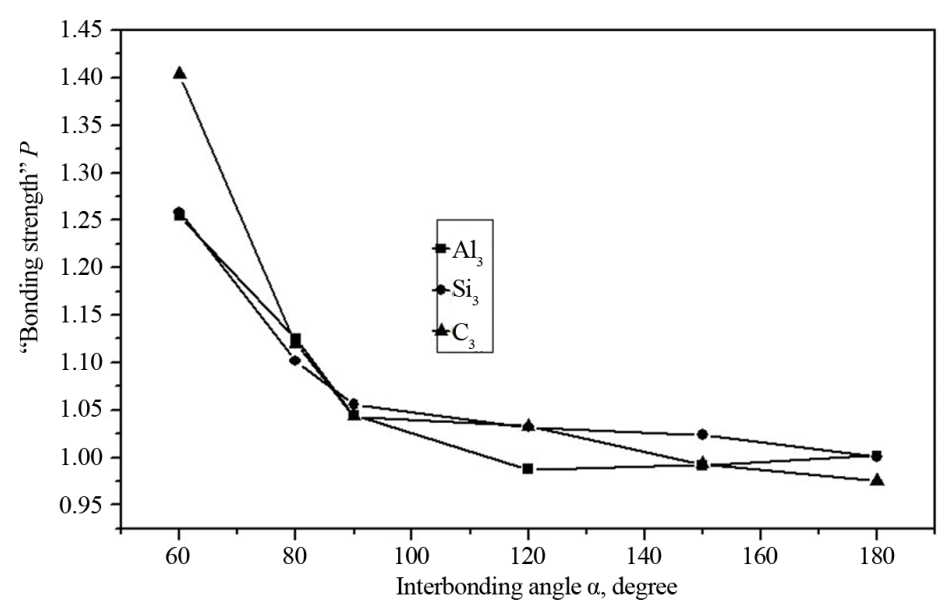

Figure 2. "The bonding strengths" in the $\mathrm{Al}_{3}, \mathrm{Si}_{3}$ and $\mathrm{C}_{3}$ trimers as functions of the corner between interatomic bonds in the case when restrictions on the interatomic electron density is absent.

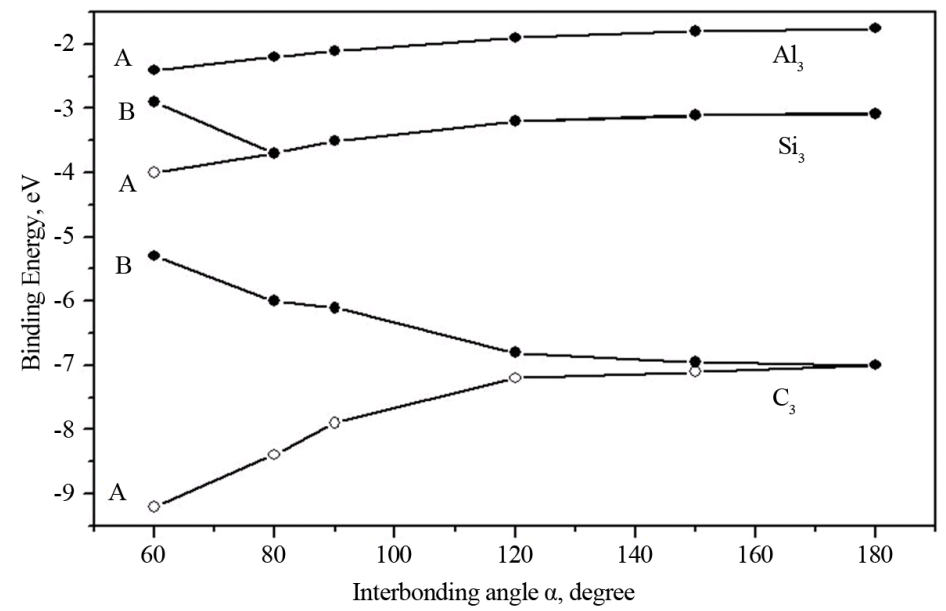

Figure 3. Calculated dependence of binding energy (on atom) for the $\mathrm{Al}_{3}, \mathrm{Si}_{3}$ and $\mathrm{C}_{3}$ trimers on the angle between interatomic bonds. A) Values are obtained without restrictions on electronic density in interatomic bonds; B) Values calculated with the condition $P=1.0$.

Table 1. Equilibrium distances $d$, angles $\alpha$ and binding energies $E_{\mathrm{b}}$ (absolute values, per atom) for $\mathrm{Si}_{3}, \mathrm{Al}_{3}$ and $\mathrm{C}_{3}$ in comparison with known calculated data.

\begin{tabular}{|c|c|c|c|c|}
\hline Trimer & Source of data & $\alpha, \operatorname{deg}$ & $d, \AA$ & $E_{b}, \mathrm{eV}$ \\
\hline & Our method & $\approx 80$ & 2.1 & 3.7 \\
\hline $\mathrm{Si}_{3}$ & Other calculations & $\begin{array}{c}77.8^{\mathrm{a}} \\
78.10^{\mathrm{c}} \\
79.6^{\mathrm{e}}\end{array}$ & $\begin{array}{c}2.26^{\mathrm{b}} \\
2.177^{\mathrm{c}}\end{array}$ & $\begin{array}{l}2.51^{\mathrm{b}} \\
2.93^{\mathrm{d}}\end{array}$ \\
\hline & Our method & 60 & 2.3 & 2.4 \\
\hline $\mathrm{Al}_{3}$ & Other calculations & $\begin{array}{l}60^{\mathrm{f}} \\
60^{\mathrm{h}}\end{array}$ & $\begin{array}{l}2.50^{\mathrm{f}} \\
2.55^{\mathrm{h}}\end{array}$ & $\begin{array}{l}1.74^{\mathrm{g}} \\
1.96^{\mathrm{f}}\end{array}$ \\
\hline & Our method & 180 & 1.2 & 7.0 \\
\hline $\mathrm{C}_{3}$ & Other calculations & $\begin{array}{l}180^{\mathrm{i}} \\
180^{\mathrm{j}}\end{array}$ & $\begin{array}{c}1.29^{\mathrm{c}} \\
1.3^{\mathrm{j}} \\
1.316^{\mathrm{k}}\end{array}$ & $\begin{array}{l}6.8^{\mathrm{i}} \\
5.0^{\mathrm{k}}\end{array}$ \\
\hline
\end{tabular}

Notations: ${ }^{\mathrm{a}}[13],{ }^{\mathrm{b}}[20],{ }^{\mathrm{c}}[21],{ }^{\mathrm{d}}[22],{ }^{\mathrm{e}}[23],{ }^{\mathrm{f}}[24],{ }^{\mathrm{g}}[15],{ }^{\mathrm{h}}[25],{ }^{\mathrm{i}}[14],{ }^{\mathrm{j}}[26],{ }^{\mathrm{k}}[27]$. 
principle) allowed us to describe correctly angular dependences of the interatomic bonding in polyatomic clusters in the framework of the orbital-free version of the density functional theory. In particular, it is possible to show that for the $\mathrm{Al}_{3}$ cluster, the equilateral triangle is favorable; the $\mathrm{Si}_{3}$ trimer is characterized by the isosceles triangle with angles of 80 and 50 degrees, and three atoms of carbon built the linear chain. Calculated equilibrium interatomic distances and the values of binding energy are well compared with the known data.

As the problem of the correct description of angles between interatomic bonds is a key point in modeling of polyatomic systems, it is possible to consider that our work opens a direct way to design an effective method of modeling of big nanosystems and supermolecules.

We have to note that the consideration which is carried out above is directly applicable only to the systems consisting of identical atoms and requires a special development for application to more complicated systems.

\section{References}

[1] Wang Y.A. and Carter E.A. (2001) Orbital-Free Kinetic-Energy Density Functional Theory. In: Schwartz, S.D., Ed., Progress in Theoretical Chemistry and Physics, Kluwer, Dordrecht, 117 p.

[2] Chen, H.J. and. Zhou, A.H. (2008) Orbital-Free Density Functional Theory for Molecular Structure Calculations. Numerical Mathematics: Theory, Methods and Applications, 1, 1-28.

[3] Zhou, B.J., Ligneres, V.L. and Carter, E.A. (2005) Improving the Orbital-Free Density Functional Theory Description of Covalent Materials. Journal Chemical Physics, 122, Article ID: 044103. http://dx.doi.org/10.1063/1.1834563

[4] Karasiev, V.V. and Trickey, S.B. (2012) Issues and Challenges in Orbital-Free Density Functional Calculations. Computational Physics Communications, 183, 2519-2527. http://dx.doi.org/10.1016/j.cpc.2012.06.016

[5] Karasiev, V.V., Chakraborty, D., Shukruto, O.A. and Trickey, S.B. (2013) Nonempirical Generalized Gradient Approximation Free-Energy Functional for Orbital-Free Simulations. Physical Review B, 88, 161108-161113(R). http://dx.doi.org/10.1103/PhysRevB.88.161108

[6] Wesolowski, T.A. (2005) Approximating the Kinetic Energy Functional Ts[p]: Lessons from Four-Electron Systems. Molecular Physics, 103, 1165-1167. http://dx.doi.org/10.1080/00268970512331339341

[7] Hung, L. and Carter, E.A. (2009) Accurate Simulations of Metals at the Mesoscale: Explicit Treatment of 1 Million Atoms with Quantum Mechanics. Chemical Physics Letters, 475, 163-170. http://dx.doi.org/10.1016/j.cplett.2009.04.059

[8] Xia, J.C., Huang, C., Shin, I. and Carter, E.A. (2012) Can Orbital-Free Density Functional Theory Simulate Molecules? The Journal of Chemical Physics, 136, Article ID: 084102(13).

[9] Lehtomäki, J., Makkonen, I., Caro, M.A., Harju, A. and Lopez-Acevedo, O. (2014) Orbital-Free Density Functional Theory Implementation with the Projector Augmented Wave Method. Journal Chemical Physics, 141, Article ID: 234102(7).

[10] Kohn, W. and Sham, J.L. (1965) Self-Consistent Equations including Exchange and Correlation Effects. Physical Review, 140, A1133-A1138. http://dx.doi.org/10.1103/physrev.140.a1133

[11] Hohenbeg, H. and Kohn, W. (1964) Inhomogeneous Electron Gas. Physical Review, 136, B864-B871. http://dx.doi.org/10.1103/PhysRev.136.B864

[12] Carling, K.M. and Carter, E.A. (2003) Orbital-Free Density Functional Theory Calculations of the Properties of Al, Mg and Al-Mg Crystalline Phases. Modeling and Simulation in Materials Science and Engineering, 11, 339-348. http://dx.doi.org/10.1088/0965-0393/11/3/307

[13] Raghavachari, K. and Logovinsky, V. (1985) Structure and Bonding in Small Silicon Clusters. Physical Review Letters, 55, 2853-2856. http://dx.doi.org/10.1103/PhysRevLett.55.2853

[14] Van Orden, A. and Saykally, R.J. (1998) Small Carbon Clusters: Spectroscopy, Structure, and Energetics. Chemical Review, 98, 2313-2357. http://dx.doi.org/10.1021/cr970086n

[15] Chuang, F.-C., Wang, C.Z. and Ho, K.H. (2006) Structure of Neutral Aluminum Clusters Aln $(2 \leq \mathrm{n} \leq 23)$ : Genetic Algorithm Tight-Binding Calculations. Physical Review B, 73, Article ID: 125431(7).

[16] Zavodinsky, V.G. and Gorkusha, O.A. (2014) Quantum-Mechanical Modeling without Wave Functions. Physics of the Solid States, 56, 2329-2335. http://dx.doi.org/10.1134/S1063783414110316

[17] Zavodinsky, V.G. and Gorkusha, O.A. (2015) New Orbital-Free Approach for Density Functional Modeling of Large Molecules and Nanoparticles. Modeling and Numerical Simulation of Material Science, 5, 39-46. http://dx.doi.org/10.4236/mnsms.2015.52004

[18] Fuchs, M. and Scheffler, M. (1999) Ab Initio Pseudopotentials for Electronic Structure Calculations of Poly-Atomic Systems Using Density-Functional Theory. Computational Physics Communications, 119, 67-98. 
http://dx.doi.org/10.1016/S0010-4655(98)00201-X

[19] Tomanek, D. and Schluter, M.A. (1987) Structure and Bonding of Small Semiconductor Clusters. Physical Review B, 36, 1208-1217. http://dx.doi.org/10.1103/PhysRevB.36.1208

[20] Mukhtarov, A.P., Normurodov, A.B., Sulaymonov, N.T. and Umarova, F.T. (2015) Charge States of Bare Silicon Clusters up to $\mathrm{Si}_{8}$ by Non-Conventional Tight-Binding Method. Journal of Nano- and Electronic Physics, 7, Article ID: 01012(7).

[21] McCarthy, M.C. and Thaddeus, P. (2003) Rotational Spectrum and Structure of $\mathrm{Si}_{3}$. Physical Review Letters, 90, Article ID: 213003(4).

[22] Liu, B., Lu, Z.Y., Pan, B., Wang, C.Z., Ho, K.M., Shvartsburg, A.A. and Jarrold, M.F. (1998) Ionization of Medium-Sized Silicon Clusters and the geometries of the Cations. Journal of Chemical Physics, 109, 9401-9409. http://dx.doi.org/10.1063/1.477601

[23] Raghavachari, K. and Rohlfing, C.M. (1988) Bonding and Stabilities of Small Silicon Clusters: A Theoretical Study of $\mathrm{Si}_{7}-\mathrm{Si}_{10}$. Journal of Chemical Physics, 89, 2219-2234. http://dx.doi.org/10.1063/1.455065

[24] Matrínez, A. and Vela, A. (1994) Stability of Charged Aluminum Clusters. Physical Review B, 49, Article ID: 17464(4).

[25] Tse, J.S. (1988) Electronic Structure of the Dimer and Trimer of Aluminium. Theoretical Chemistry (Journal of Molecular Structures), 165, 21-24.

[26] Karton, A., Tarnopolsky, A. and Martin, J.M.L. (2009) Atomization Energies of the Carbon Clusters $C_{n}(n=2-10)$ Revisited by Means of W4 Theory as Well as Density Functional, Gn, and CBS Methods. International Journal of Interface between Chemistry and Physics, 107, 977-1003. http://dx.doi.org/10.1080/00268970802708959

[27] Afshar, M., Babaei, M. and Kordbacheh, A.H. (2014) First Principles Study on Structural and Magnetic Properties of Small and Pure Carbon Clusters $\left(\mathrm{C}_{\mathrm{n}}, \mathrm{n}=2\right.$ - 12). Journal of Theoretical and Applied Physics, 8, 103-108. http://dx.doi.org/10.1007/s40094-014-0136-6

[28] Beckstedte, M., Kley, A., Neugebauer, J. and Scheffler, M. (1997) Density Functional Theory Calculation for Poly-Atomic Systems: Electronic Structure, Static and Elastic Properties and ab Initio Molecular Dynamics. Computational Physics Communications, 107, 187-205. http://dx.doi.org/10.1016/S0010-4655(97)00117-3 